\title{
Review of lung sealant technologies for lung volume reduction in pulmonary disease
}

This article was published in the following Dove Press journal:

Medical Devices: Evidence and Research

\section{Branislava Milenkovic ${ }^{1,2}$ Sanja Dimic Janjic ${ }^{2}$ Spasoje Popevic ${ }^{1,2}$}

'Faculty of Medicine, University of Belgrade, Belgrade, Serbia; ${ }^{2} \mathrm{Clinic}$ for Pulmonary Diseases, Clinical Center of Serbia, Belgrade, Serbia
Correspondence: Branislava Milenkovic Clinic for Pulmonary Diseases, Clinical Center of Serbia, Koste Todorovica 26, I 1000 Belgrade, Serbia

Tel/fax +38I II 3663267

Email branislava.milenkovic@kcs.ac.rs

\begin{abstract}
Emphysema is an incurable and underdiagnosed disease with obstructive ventilatory impairment of lung function. Despite decades of research, medical treatments available so far did not significantly improve the survival benefits. Different bronchoscopic methods for lung volume reduction (LVR) in emphysema were used in the past 2 decades aiming to close the airways serving the hyperinflated lung regions and to allow the gas in the more distal bullas to be absorbed. Sealants and adhesives can be natural/biological, synthetic and semisynthetic. In lung surgery, lung sealants are used to treat prolonged air leak, which is the most common complication. Sealants can also be applied in bronchoscopic lung volume reduction (BLVR) as they administer into the peripheral airways where they polymerize and act as tissue glue on the surface of the lung to seal the target area to cause durable permanent absorption atelectasis. Initial studies analyzed the efficacy of bronchoscopic instillation of a fibrinogen-thrombin complex solution in advanced emphysema. Future studies will analyze the effects of adding chondroitin sulfate and poly-L-lysine to thrombin-fibrinogen complex thus promoting fibroblast attachment, proliferation and scarring, causing bronchial fibrostenosis and preventing ventilation of the affected part of the lung. Modifications of these methods were later developed, and the efficacy of BLVR with other sealants was analyzed in clinical studies. Results from current studies using this treatment method are promising showing that it is effective in improving exercise tolerance and quality of life in patients with advanced emphysema. It seems that subjective benefits in dyspnea scores and quality of life are more marked than improvements in lung function tests. The safety profile of sealant techniques in BLVR was mostly acceptable in clinical studies. The definite conclusions about the effectiveness of sealant in BLVR could be difficult because only a small population was involved in the current studies. More randomized large controlled studies are needed in establishing the definite role of biological BLVR in the bronchoscopic treatment of emphysema. Keywords: sealant, emphysema, lung volume reduction
\end{abstract}

\section{Introduction to current management strategies for patients with emphysema}

Emphysema is an incurable and underdiagnosed disease with irreversible enlargement of alveolar spaces distal to the terminal bronchiole. The number and size of alveolar fenestrae are increased with eventual destruction of alveolar septa (Figure 1). Centriacinar emphysema affects the respiratory bronchioles, panacinar emphysema affects central and peripheral portions of acinus, and periacinar/paraseptal emphysema is characterized with distention of alveolar spaces adjacent to septal and pleural surfaces. ${ }^{1}$ Alveoli and alveolar ducts can be enlarged in people older than 50 years with 
senile emphysema. Destruction of alveoli results in cysts or bullae that increase physiological dead space and compress the surrounding lung tissue. Hogg et $\mathrm{al}^{1}$ measured the extent of emphysema, the number of terminal bronchioles and the minimum diameters and cross-sectional areas of terminal bronchioles in isolated lungs removed from patients with COPD who underwent lung transplantation and in donor (control) lungs using multidetector computed tomography (CT). Their results suggested that narrowing and disappearance of small conducting airways before the onset of emphysematous destruction can explain the increased peripheral airway resistance reported in COPD. ${ }^{1}$

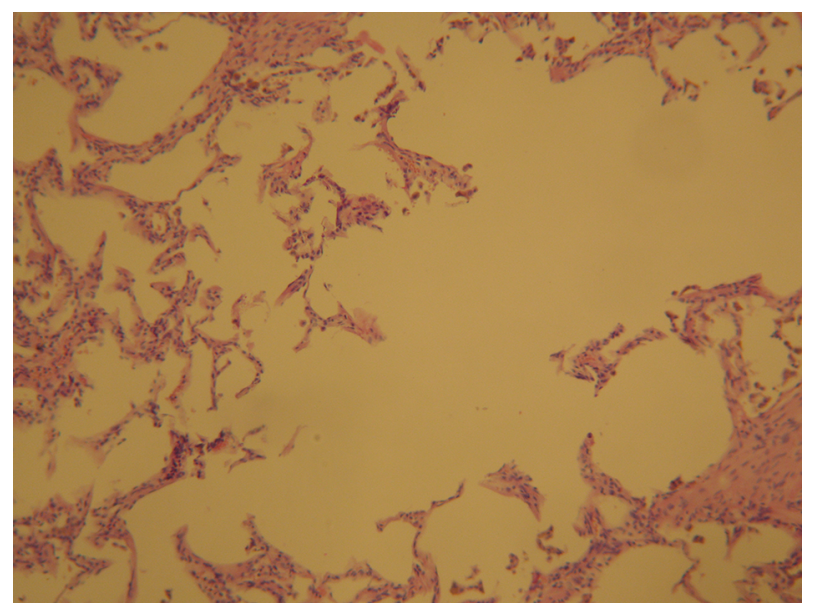

Figure I Photomicrographs of emphysematous lung parenchyma showing hyperdistension of alveolar ducts, increased number and size of alveolar fenestrae with marked destruction of alveolar septa $(\mathrm{H} \& \mathrm{E}) \times 100$ increased (by courtesy of Dr Jelena Stojsic).
The destruction of lung tissue can be visualized on highresolution CT (HRCT; Figure 2).

Emphysema is recognized as a phenotype of COPD, and it is found in most patients with COPD. The main symptom is progressive dyspnea during exercise. Patients with emphysema tend to be very symptomatic with shortness of breath even on mild exertion. Dyspnea is largely owing to hyperinflation in emphysema., ${ }^{2,3}$ Lung function in patients with emphysema is abnormal with obstructive ventilatory impairment. Destruction of alveoli with cysts or bullae does not contribute to gas exchange. Lung density can be assessed using CT analyzing attenuation of the pulmonary tissue which reflects the degree of structural lung abnormalities (Figure 3). ${ }^{4}$

Despite decades of research, medical treatments available so far have helped improve quality of life but did not significantly improve the survival benefits. Unfortunately, the drug that can repair lung tissue is not yet available. Nonpharmacological treatment such as smoking cessation and respiratory rehabilitation has beneficial effects on patient's lung function, exercise tolerance, quality of life and overall survival. Bronchodilator therapy is mostly ineffective because the cysts and bullae tend to increase in size and number over time and occupy space and press the surrounding lung tissue.

Surgical (nonbronchoscopic) lung volume resection is performed in patients with emphysema to achieve expansion of healthy lung tissue that surrounds giant bullae. Bullectomy can be performed using two surgical approaches

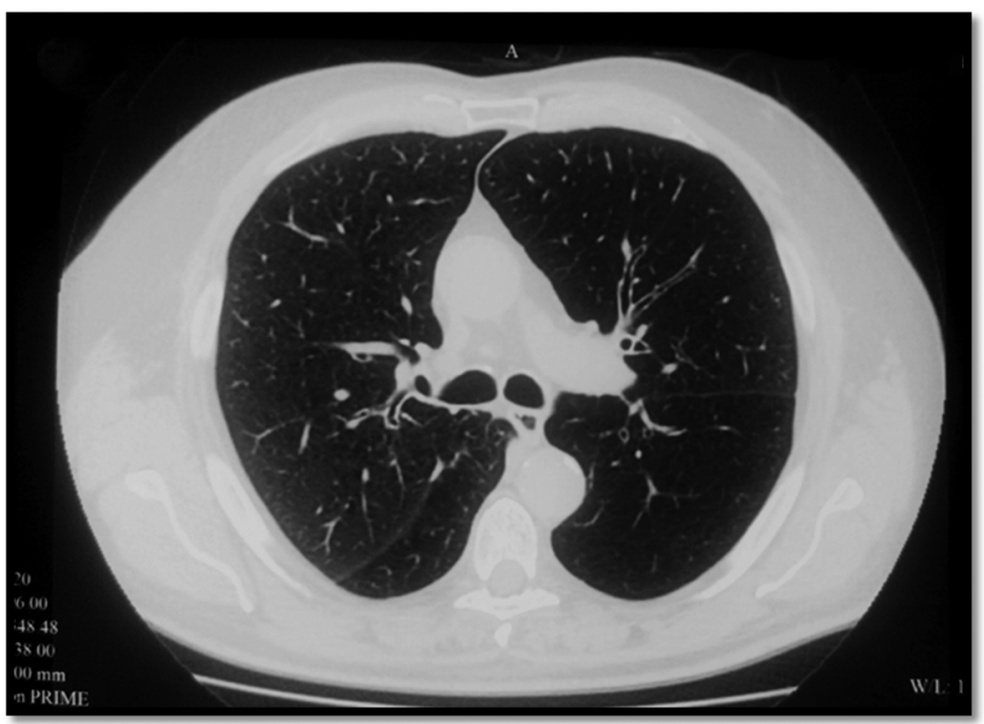

Figure $2 \mathrm{CT}$ of a patient with emphysema with giant bulla.

Note: Chest HRCT of 7I-year-old man with stable COPD (GOLD III) showing multiple areas of decreased attenuation bilaterally, (hematoxylin-eosin, original magnification $\times 100)$.

Abbreviations: CT, computed tomography; HRCT, high-resolution CT; GOLD, Global Initiative on Obstructive Lung Disease. 


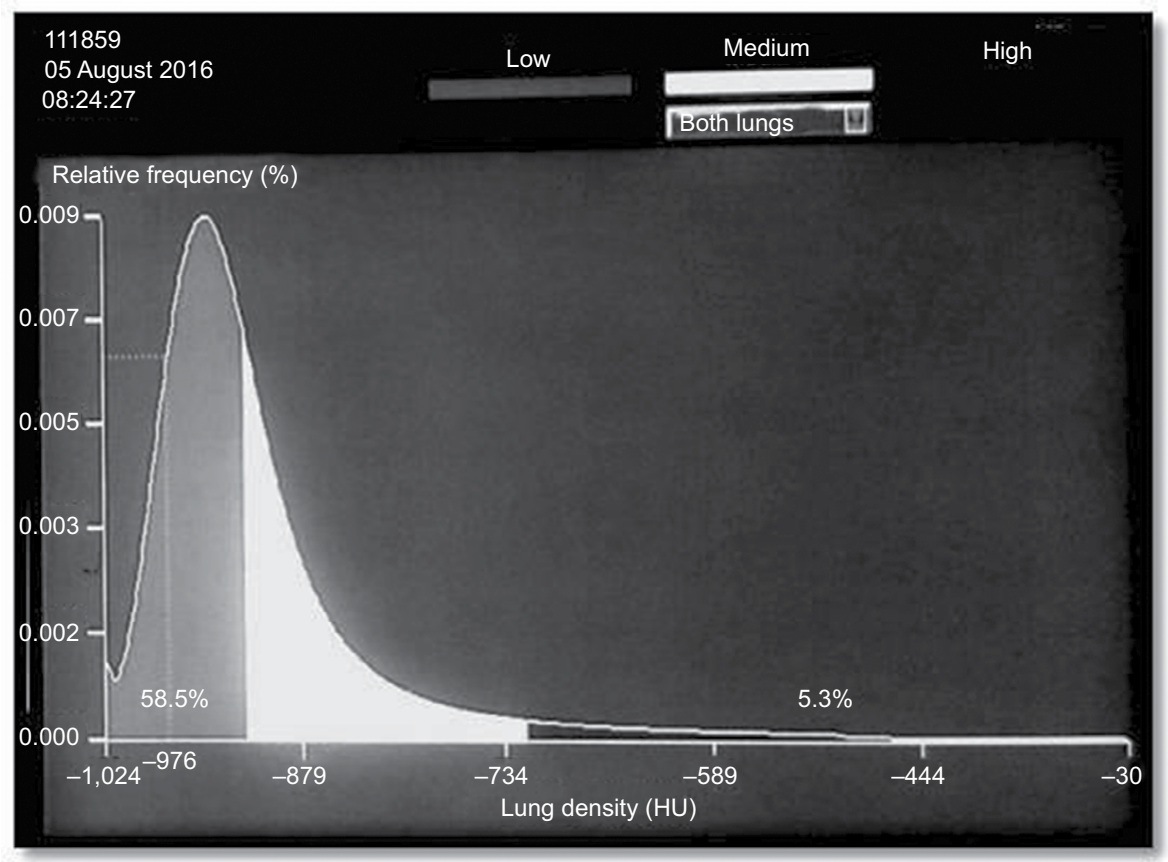

Figure 3 Lung density histogram in 7I-year-old man with stable COPD (GOLD III). Note: Lung density index was $58.0 \%$ for right lung and $62.7 \%$ for left lung. Abbreviation: GOLD, Global Initiative on Obstructive Lung Disease.

such as thoracotomy/sternotomy and video-assisted thoracic surgery (VATS). Removing the giant bulla improves the chest mechanics and remodels the thorax and diaphragm, with symptomatic and functional improvement. VATS was transpired as a valuable surgical option that allows quicker recovery and is associated with less pain and with lower morbidity and mortality than open thoracotomy. ${ }^{5,6}$ Nevertheless, the overall mortality over 29-month follow-up was same in the lung volume reduction surgery (LVRS) and medically treated groups, and the overall risk of death in the first 3 months was higher in the LVRS group than the medical group. ${ }^{7}$ Upper lobe emphysema has been associated with short-term improvement in pulmonary function after LVRS, and by NETT trial, and is predictive of improved survival in patients who also have low maximal exercise capacity. It further implies that upper lobe predominance, as compared to other patterns, may result in clearer and more accessible areas for excision, or that lungs in other areas are healthier. ${ }^{8}$

The common LVRS-related morbidity has led to the development of bronchoscopic lung volume reduction (BLVR). BLVR refers to several bronchoscopic techniques for treating severe emphysema such as bronchial valves, bronchial plugs/occluders/blockers, biological BLVR
(Bio-BLVR), bronchoscopic thermal vapor ablation, endobronchial coils, bronchial fenestration and airway bypass and some techniques that are analyzed in clinical experimentation. ${ }^{9-14}$

Several ways of closing the airways that served the hyperinflated regions have been attempted with the primary aim to allow the gas in the more distal bullae to be reabsorbed over the next days and weeks. Lung sealant technologies include instillation of biologically active reagents as tissue sealants that lead to replacement of diseased emphysematous tissue by a contracted organized scar thus making it essentially irreversible procedure. Lung sealant is injected into the peripheral airways where it polymerizes and acts as tissue glue on the surface of the lung to seal the target area to cause durable permanent absorption atelectasis. ${ }^{15}$

\section{Efficacy studies including relevant randomized and case studies of sealants in respiratory medicine Review of clinical utility of sealants in thoracic surgery}

Sealants can be used for sutures to establish tissue continuity instead of traditional surgical closure. In the past decade, 
tissue defects were treated with sutures, wires and staples, and bleeding was controlled with cautery. Nowadays, adhesives and sealants are finding their place in surgery with different uses along with different advantages and disadvantages. Sealants and adhesives can be natural/biological (polypeptide/ protein-based and polysaccharide-based sealants), synthetic and semisynthetic. Concerning the indication field, the surgical sealants are used for hemostasis, tissue sealing and tissue engineering and are applicable in several body tissues. Challenge of sealant application differ among the different body structures, since some of the organs are more complex due to their consistency and biological characteristics.

In lung surgery, lung tissues can be sealed using mechanical methods such as sutures, staples or the implantation of surgical meshes. These methods are inevitably associated with lung tissue damage caused by deep piercing, ischemia and prolonged air leaks. ${ }^{16}$ Prolonged air leak represents the most common complication after lung surgery and could lead to extended chest tube drainage time and development of infections, and broncho-pleural fistulae..$^{5,17,18}$

In addition, lung tissues can be sealed biologically via lung sealants/adhesives. Lung sealants must demonstrate specific characteristics due to the morphologic, functional and immunologic organ complexity. Respiratory movements pose additional stress to the reparation site; therefore, strong adhesiveness to the tissue is desirable. However, to preserve elastic recoil of the lung, sealant/adhesive should be highly elastic and flexible, as well. ${ }^{19}$ Nonsterile and potentially septic environment in lungs can predispose wound infection. ${ }^{20}$ Lungs are very immunogenic, so sealant should be biocompatible and biodegradable to avoid inflammation. ${ }^{21}$ Potential air or liquid leakages during lung surgery necessitate sealants that tolerate high pressure and wet environment. ${ }^{22}$ Concerning the complex vascularity of the lungs, sealant/adhesive should also have the function of hemostasis. Limited space sometimes complicates suturing, so sealant/adhesive can be delivered through applicators; in addition, endoscopic procedures can be a possible method to deliver sealant.

\section{Review of clinical utility of sealants in bronchoscopic volume reduction methods}

Biological lung volume reduction (Bio-LVR) uses direct application of a sealant or remodeling substances aimed at obtaining atelectasis and fibrosis of the lung parenchyma, thus preventing ventilation of these parts. Initial human studies were using bronchoscopic instillation of a fibrinogenthrombin complex solution. ${ }^{23,24}$ Modification of that method, called Bio-LVR, was developed, adding chondroitin sulfate and poly-L-lysine to thrombin-fibrinogen complex. The idea was to form a hydrogel which can promote fibroblast attachment, proliferation and scarring, cause bronchial fibrostenosis and prevent ventilation of the affected part of the lung. Gel polymerization occurred in situ, which produced localized inflammatory reaction and resorptive atelectasis and lung region collapse over 4-6 weeks. ${ }^{24,25}$ Hydrogel was also used to seal interalveolar and bronchiole-alveolar pores which was supposed to result in disabling collateral ventilation.

The procedure is performed under conscious sedation using flexible bronchoscope which is introduced and wedged into the segmental or subsegmental airway leading to emphysematous part of the lung. To collapse distal airways, suction is applied through the bronchoscope. First, the enzymatic solution (eg, porcine trypsin) is instilled into the airway to detach epithelial cells from bronchial wall and to prepare mucosa for fibroblast adherence. After 2 minutes, that solution had to be washed out and a dual lumen catheter with thrombin and fibrinogen placed through the bronchoscope. The contents are instilled and pushed distally, mixing in the distal airway. The liquid component must fill the alveoli and block collateral ventilation. Each subsegmental application takes $\sim 10$ minutes and four to eight subsegments are treated during a single procedure. ${ }^{28}$

This method was partially successful in the study of patients affected by heterogeneous, predominant upper lobe emphysema, showing mild-to-moderate improvement (improvement in pulmonary function, better dyspnea scores and quality of life), which lasted up to 6 months. ${ }^{26}$ Similar results were reported in a study of 25 patients with bilateral homogeneous emphysema in whom high- or low-dose hydrogel was administered to eight subsegments. ${ }^{13}$

Another observational study compared the effect of single lobe versus scattered double lobe Bio-LVR for predominant upper lobe emphysema. ${ }^{28}$ Single lobe treatment led to a greater improvement in forced expiratory volume in 1 second $\left(\mathrm{FEV}_{1}\right)$ at 12 weeks after the procedure compared to scattered double lobe approach. It can be explained by reducing collateral ventilation to other lobes by performing complete treatment of only one lobe. ${ }^{27}$

There is also an alternative method that uses a synthetic polymeric foam called emphysematous lung sealant (ELS) with similar application technique. Herth et $\mathrm{al}^{29}$ performed initial tests using ELS (AeriSeal ${ }^{\circledR}$ System; Pulmonx Corporation, Redwood City, CA, USA) in 25 patients with heterogeneous emphysema in 2011. There was an improvement in some lung function parameters (increased $\mathrm{FEV}_{1}, \mathrm{FVC}$, 
6-minute walk test and decreased residual volume $[\mathrm{RV}] /$ total lung capacity [TLC]), but only the improvement in FVC was statistically significant. Results were better in 14 patients in Global Initiative on Obstructive Lung Disease (GOLD) stage III compared with 11 subjects in GOLD stage IV for whom the benefit was less relevant. ${ }^{29,30}$ Over $90 \%$ of patients treated with Bio-LVR experienced flu-like symptoms, such as fever, dyspnea, pleurisy, nausea, headache and leukocytosis, within 24 hours of the procedure. These symptoms resolved in 24-48 hours. ${ }^{27,29}$

Magnussen et a ${ }^{31}$ investigated the effect of interlobar fissure integrity on responses to treatment with an endoscopic tissue sealant (AeriSeal ELS) that collapses hyperinflated lung. They demonstrated that fissure integrity did not contribute to posttreatment changes in $\mathrm{FEV}_{1}$, RV/TLC ratio or lobar volume measured by $\mathrm{CT}$ analysis in patients with severe upper lobe predominant emphysema.

Safety and efficacy of ELS were assessed in 57 patients who were randomly assigned to two groups: the first group had ELS (two subsegments in each upper lobe) and received medical therapy and the second group had medical therapy alone. ${ }^{31}$ Significant improvements were noted in lung function, dyspnea and quality of life in the ELS and medical therapy groups, and benefits persisted for 6 months. However, serious adverse events requiring hospitalization occurred in $44 \%$ of patients. Treatment responders tended to be those experiencing respiratory adverse events. ${ }^{32}$

Recent study showed promising results in maintaining the improvement of volumetric $\mathrm{HRCT}$ and $\mathrm{FEV}_{1} / \mathrm{FVC}$ ratio. ${ }^{33} \mathrm{It}$ was performed in 15 patients in which autologous blood and fibrin glue were used to achieve BLVR and showed promising results in maintaining HRCT volumetry improvement and $\mathrm{FEV}_{1} / \mathrm{FVC}$ ratio over 12-week assessment period. Within both groups, there was statistically significant improvement in dyspnea, quality of life and exercise tolerance at 12 weeks postprocedure compared with baseline value. ${ }^{33}$

Kramer et al, assessed the safety and efficacy of bilateral AeriSeal Emphysematous Lung Sealant System (ELS) in the treatment of patients with advanced emphysema. The study demonstrated short procedure time and length of hospital stay (average 1.1 day), successful primary end point of a reduction at 3 months in upper lobe lung volume analyzed by quantitative CT scan, with improvements in spirometry, gas trapping, diffusing capacity of lung for carbon monoxide, symptom scores (modified Medical Research Council dyspnea score) and health-related quality of life (St. George Respiratory Questionnaire) measured at 6 and 12 months. In the further studies, Kramer et al ${ }^{35}$ summarized the safety and efficacy data of patients from the initial ELS study after 2 years and suggested that the beneficial effects of lung sealant therapy, previously reported at 1 year after treatment, persist to 2 years and beyond with a favorable long-term safety profile.

Fruchter et $\mathrm{a}^{36}$ demonstrated that previous BLVR treatment was not associated with different outcomes following lung transplantation, but with evident increased bacterial colonization rates. However, when investigating correlation among airway bacterial colonization $(\mathrm{ABC})$, serum C-reactive protein (CRP) level and the risk of COPD exacerbation within 1 month following BLVR, Fruchter et $\mathrm{al}^{37}$ demonstrated that $\mathrm{ABC}$ is common in severe COPD patients undergoing BLVR, and along with elevated CRP level they are associated with high risk of immediate postprocedural COPD exacerbation.

Common inclusion and exclusion criteria for BLVR are summarized in Table 1. Patients with heterogenous "scattered" distribution of emphysema are not candidates for BLVR. Patients with heterogenous emphysema and COPD, GOLD stage III, had better treatment results.

The safety of Bio-BLVR was analyzed in clinical studies. There were no treatment-related deaths, pulmonary emboli, episodes of heart failure, cardiac ischemia or myocardial infarction or severe cardiac arrhythmia. In addition, there were no serious procedural or immediate postprocedural complications, pneumothorax, bleeding episodes, respiratory failure requiring ventilator support, empyemas and lung abscesses. A few patients experienced spillage of material from the administration site into the central airways, but the material was cleared safely by suctioning through the bronchoscope in all patients.

However, BLVR treatment was associated with significant side effects. All patients who underwent BLVR experienced a transient inflammatory reaction, ie, "flu-like" reaction characterized by leukocytosis, elevated sedimentation rate and/ or CRP, fever and malaise within 8-24 hours of treatment. In most cases, this reaction was self-limited and resolved

Table I Common inclusion and exclusion criteria for Bio$\mathrm{BLVR}^{2,11,25,28,9}$

\begin{tabular}{ll}
\hline $\begin{array}{l}\text { Inclusion criteria for } \\
\text { Bio-BLVR }\end{array}$ & $\begin{array}{l}\text { Relative exclusion criteria for } \\
\text { Bio-BLVR }\end{array}$ \\
\hline $\begin{array}{l}\text { Advanced single (upper) lobe } \\
\text { predominant emphysema }\end{array}$ & $\begin{array}{l}\text { Heterogenous "scattered } \\
\text { distribution" of emphysema } \\
\text { Homogenous emphysema" }\end{array}$ \\
$\begin{array}{l}\text { Absence of clinically significant } \\
\text { pulmonary hypertension }\end{array}$ \\
Heterogenous emphysema & \\
(GOLD stage III) $^{29}$ & \\
\hline
\end{tabular}

Abbreviations: Bio-BLVR, biological bronchoscopic ${ }^{29}$ lung volume reduction GOLD, Global Initiative on Obstructive Lung Disease. 
within 24-96 hours with supportive care (nonsteroidal antiinflammatory medications, acetaminophen, corticosteroids, bronchodilators and antibiotics as indicated). Some of these patients experienced COPD exacerbation, and some of them were related to the treatment procedure.

Although the study data showed that BLVR is a potential alternative to LVRS, questions about the techniques arise, such as how to prevent and reduce the treatment-associated inflammatory response, to predict the clinical outcome and to maintain the long-term efficacy with promising safety data.

\section{Conclusion and future perspectives}

The currently available data on the efficacy of numerous Bio-BLVR methods are not consistently conclusive.

Lung sealant technology is a novel and an effective approach for BLVR in patients with advanced emphysema. The results of the current studies about this treatment method are promising because they demonstrated bronchoscopic administration of sealants to be an effective method of improving respiratory function, exercise tolerance and quality of life in patients with advanced emphysema. It seems that subjective benefits in dyspnea scores and quality of life are more marked than improvements in lung function tests.

It is difficult to make the definite conclusion about the effectiveness of bronchoscopic administration of lung sealants LVRS due to small patient populations analyzed in current studies. More randomized large controlled studies are required in establishing the role of Bio-LVR in the treatment of emphysema, especially in the light of current achievements in emphysema phenotyping.

\section{Disclosure}

The authors report no conflicts of interest in this work.

\section{References}

1. Hogg JC, Senior RM. Chronic obstructive pulmonary disease - part 2: pathology and biochemistry of emphysema. Thorax. 2002;57(9):830-834.

2. McDonough JE, Yuan R, Suzuki M, et al. Small-airway obstruction and emphysema in chronic obstructive pulmonary disease. $N$ Engl $\mathrm{J}$ Med. 2011;365(17):1567-1575.

3. Gagnon P, Guenette JA, Langer D, et al. Pathogenesis of hyperinflation in chronic obstructive pulmonary disease. Int J Chron Obstruct Pulmon Dis. 2014;9:187-201.

4. Mascalchi M, Camiciottoli G, Diciotti S. Lung densitometry: why, how and when. J Thorac Dis. 2017;9(9):3319-3345.

5. Armstrong HF, Dussault NE, Thirapatarapong W, Lemieux RS, Thomashow BM, Bartels MN. Ventilatory efficiency before and after lung volume reduction surgery. Respir Care. 2015;60(1):63-71.

6. O'Brien GM, Criner GJ. Surgery for severe COPD and lung volume reduction for emphysema. Chest. 2004;126:238-248.
7. Fishman A, Martinez F, Naunheim K, et al. A randomized trial comparing lung-volume-reduction surgery with medical therapy for severe emphysema. N Engl J Med. 2003;348(21):2059-2073.

8. National Emphysema Treatment Trial Research Group. A randomized trial comparing lung-volume reduction surgery with medical therapy for severe emphysema. N Engl J Med. 2003;348(21):2059-2073.

9. Valipour A, Herth FJF, Burghuber OC, et al. Target lobe volume reduction and COPD outcome measures after endobronchial valve therapy. Eur Respir J. 2014;43(2):387-396.

10. Ingenito EP, Berger RL, Henderson AC, Reilly JJ, Tsai L, Hoffman A. Bronchoscopic lung volume reduction using tissue engineering principles. Am J Respir Crit Care Med. 2003;167(5):771-778.

11. Refaely Y, Dransfield M, Kramer MR, et al. Biologic lung volume reduction therapy for advanced homogeneous emphysema. Eur Respir J. 2010;36(1):20-27.

12. Shah PL, Gompelmann D, Valipour A, et al. Thermal vapour ablation to reduce segmental volume in patients with severe emphysema: STEP-UP 12 month results. Lancet Respir Med. 2016;4(9):e44-e45.

13. Sciurba FC, Criner GJ, Strange C, et al; RENEW Study Research Group. Effect of endobronchial coils vs usual care on exercise tolerance in patients with severe emphysema: the RENEW Randomized Clinical Trial. JAMA. 2016;315(20):2178-2189.

14. Cardoso PFG, Snell GI, Hopkins P, et al. Clinical application of airway bypass with paclitaxel-eluting stents: early results. J Thorac Cardiovasc Surg. 2007;134(4):974-981.

15. Falkenstern-Ge RF, Ingerl H, Kohlhäufl M. Severe emphysema treated by endoscopic bronchial volume reduction with lung sealant (AeriSeal). Case Rep Pulmonol. 2013;2013:361391.

16. Belboul A, Dernevik L, Aljassim O, Skrbic B, Radberg G, Roberts D. The effect of autologous fibrin sealant (Vivostat) on morbidity after pulmonary lobectomy: a prospective randomised, blinded study. Eur $J$ Cardiothorac Surg. 2004;26(6):1187-1191.

17. D'Andrilli A, Andreetti C, Ibrahim M, et al. A prospective randomized study to assess the efficacy of a surgical sealant to treat air leaks in lung surgery. Eur J Cardiothorac Surg. 2009;35(5):817-820.

18. Bertolaccini L, Lyberis P, Manno E. Lung sealant and morbidity after pleural decortication: a prospective randomized, blinded study. J Cardiothorac Surg. 2010;5:45.

19. Annabi N, Yue K, Tamayol A, Khademhosseini A. Elastic sealants for surgical applications. Eur J Pharm Biopharm. 2015;95(Pt A):27-39.

20. Montanaro L, Arciola CR, Cenni E, et al. Cytotoxicity, blood compatibility and antimicrobial activity of two cyanoacrylate glues for surgical use. Biomaterials. 2001;22(1):59-66.

21. Assmann A, Vegh A, Ghasemi-Rad M, et al. A highly adhesive and naturally derived sealant. Biomaterials. 2017;140:115-127.

22. Itano $\mathrm{H}$. The optimal technique for combined application of fibrin sealant and bioabsorbable felt against alveolar air leakage. Eur J Cardiothorac Surg. 2008;33(3):457-460.

23. Hillerdal G, Gustafsson G, Wegenius G, Englesson S, Hedenström H, Hedenstierna G. Large emphysematous bullae. Successful treatment with thoracoscopic technique using fibrin glue in poor-risk patients. Chest. 1995;107(5):1450-1453.

24. Reilly J, Washko G, Pinto-Plata V, et al. Biological lung volume reduction: a new bronchoscopic therapy for advanced emphysema. Chest. 2007;131(4):1108-1113.

25. Criner GJ, Pinto-Plata V, Strange C, et al. Biologic lung volume reduction in advanced upper lobe emphysema: phase 2 results. Am J Respir Crit Care Med. 2009;179(9):791-798.

26. Ernst A, Anantham D. Endoscopic management of emphysema. Clin Chest Med. 2010;31(1):117-126.

27. Berger RL, Decamp MM, Criner GJ, Celli BR. Lung volume reduction therapies for advanced emphysema: an update. Chest. 2010;138(2):407-417.

28. Strange C, Criner G, Leeds W, et al. Improved efficacy of biological lung volume reduction (BLVR) therapy with lobar targeting [abstract]. Am J Respir Crit Care Med. 2009;179:A4391. 
29. Herth FJ, Gompelmann D, Stanzel F, et al. Treatment of advanced

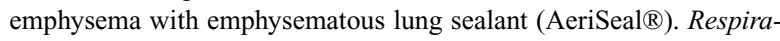
tion. 2011;82(1):36-45.

30. Global Initiative for Chronic Obstructive Lung Disease (GOLD) [homepage on the Internet]. Global Strategy for the Diagnosis, Management, and Prevention of Chronic Obstructive Pulmonary Disease. 2017. Available from: www.goldcopd.org. Accessed April 15, 2017.

31. Magnussen H, Kramer MR, Kirsten AM, et al. Effect of fissure integrity on lung volume reduction using a polymer sealant in advanced emphysema. Thorax. 2012;67(4):302-308.

32. Come CE, Kramer MR, Dransfield MT, et al. A randomised trial of lung sealant versus medical therapy for advanced emphysema. Eur Respir J. 2015;46(3):651-662.

33. Bakeer M,AbdelgawadTT, El-Metwaly R, El-Morsi A, El-Badrawy MK, ElSharawy S. Low cost biological lung volume reduction therapy for advanced emphysema. Int J Chron Obstruct Pulmon Dis. 2016;11:1793-1800.
34. Kramer MR, Refaely Y, Maimon N, Rosengarten D, Fruchter O. Bilateral endoscopic sealant lung volume reduction therapy for advanced emphysema. Chest. 2012;142(5):1111-1117.

35. Kramer MR, Refaely Y, Maimon N, Rosengarten D, Fruchter O. Two-year follow-up in patients treated with emphysematous lung sealant for advanced emphysema. Chest. 2013;144(5): $1677-1680$.

36. Fruchter O, Fridel L, Kramer MR. The pathological features of bronchoscopic lung volume reduction using sealant treatment assessed in lung explants of patients who underwent lung transplantation. Respiration. 2013;86(2):143-144.

37. Fruchter O, Rosengarten D, Goldberg E, Ben-Zvi H, Tor R, Kramer MR. Airway bacterial colonization and serum C-reactive protein are associated with chronic obstructive pulmonary disease exacerbation following bronchoscopic lung volume reduction. Clin Respir J. 2016;10(2):239-245.
Medical Devices: Evidence and Research

\section{Publish your work in this journal}

Medical Devices: Evidence and Research is an international, peerreviewed, open access journal that focuses on the evidence, technology, research, and expert opinion supporting the use and application of medical devices in the diagnosis, monitoring, treatment and management of clinical conditions and physiological processes. The identification of novel

\section{Dovepress}

devices and optimal use of existing devices which will lead to improved clinical outcomes and more effective patient management and safety is a key feature. The manuscript management system is completely online and includes a quick and fair peer-review system. Visit http://www. dovepress.com/testimonials.php to read real quotes from authors.

Submit your manuscript here: https://www.dovepress.com/medical-devices-evidence-and-research-journal 